\title{
NADA EN LA EDUCACIÓN ESTÁ PERDIDO
}

\section{Entrevista a César Cortez Mondragón}

\author{
Janina Navarro Linares* \\ Universidad Católica Sedes Sapientiae \\ jnavarro@ucss.edu.pe
}

Resumen: Ayudante de una farmacia, educador, historiador, director de colegio, instituto y director general de Educación Secundaria en el Minedu; también, autor de libros sobre historia, economía, educación, psicología, entre otros. A través de sus palabras, el profesor César Cortez Mondragón (Chiclayo, 1940) logra expresar una gran pasión y esperanza por la educación peruana. En este diálogo nos compartirá, además, facetas de su vida, experiencia y sabiduría para nuestras generaciones.

Palabras clave: Historia, educación, realidad peruana, gestión educativa.

* Janina Navarro Linares es licenciada en Educación por la Universidad Católica Sedes Sapientiae con un diplomado en Doctrina Social de la Iglesia. Es candidata a magíster en Filosofía por la misma universidad. Asimismo, ha publicado en la revista Studium Veritatis artículos de investigación sobre ciencia y religión y ética educativa. Del mismo modo, ha ejercido el cargo de coordinadora académica en la UCSS del proyecto internacional "La vida en el universo: su origen, su naturaleza, su sentido" auspiciado por la Universidad de Oxford y la Fundación Templeton, durante el 2016 y 2017, respectivamente. Actualmente, dicta los cursos de Filosofía, Epistemología y Antropología Religiosa en la unidad de pregrado de las facultades de Educación. Ciencias de la Salud, Derecho, Ciencias Económicas e Ingeniería de la UCSS. 


\title{
NOTHING IN EDUCATION IS LOST INTERVIEW WITH CÉSAR CORTEZ MONDRAGÓN
}

\begin{abstract}
Assistant of a pharmacy, educator, historian, director of school, institute and general director of Secondary Education in Minedu; also, author of history, economics, education, psychology, history, among others. Through his words, Professor César Cortez Mondragón (Chiclayo, 1940) manages to express a great passion and hope for Peruvian education. In this dialogue, he will also share facets of his life, experience and wisdom for our generations.
\end{abstract} KeYwords: History, education, Peruvian reality, educational management.

\section{Profesor, usted nació en Lambayeque, ¿qué recuerdos significativos tiene de su tierra natal?}

Mira, yo nací en un distrito que se llama Zaña. La familia de mi mamá era de un distrito de Cajamarca y, por mi papá, de la provincia de Pacasmayo. Se conocieron en Zaña, donde trabajaba mi papá. Yo viví allá hasta los siete años. Esta ciudad se fundó en el siglo XVI y en ella vivió, por ese entonces, la aristocracia espańola. Aunque también, por aquel tiempo, el Fenómeno del Niño arrasó con todo y ellos huyeron. Dejaron a los esclavos negros y la ciudad se quedó con una rica cultura negra, muy parecida a Chincha. Lo simpático era que mi familia no tenía nada de la cultura negra, pero yo sí asimilé mucho de ella.

\section{¿Cómo qué?}

Por ejemplo, la veneración a santo Toribio, quien murió en Zaña durante una visita pastoral. Eso le llamó mucho la atención a... 


\section{¿Al profesor José Antonio Benito?}

Sí, porque ahí en Zaña hay un instituto que se llama Santo Toribio. El profesor Benito, quien conocía la vida del santo, me animó para conocer Mayorga (España), la tierra del obispo Toribio de Mogrovejo, pero esa es otra historia... (Risas). Después, con mi familia nos mudamos a un distrito de Pacasmayo, pero cada vez que se celebraba la fiesta de santo Toribio, me llevaban de nuevo (a Zaña).

\section{¿Qué tan significativo era para ese pueblo dicha fiesta?}

Es que allí murió santo Toribio, por eso es el santo patrón de Zaña. Otra cosa que me acuerdo mucho eran las fiestas en su honor, había mucha música negra y me identificaba con ella.

\section{Aunque no la bailaba...}

Pues no, (risas), pero me gustaba escucharla. También me gustaba mucho escuchar a los decimistas que venían para la fiesta y se retaban con guitarra en la mano o con instrumentos musicales como checos (instrumentos de calabazas), troncos de madera y tocaban con ellas.

¿A qué se dedicaba su familia?, ¿influyó de algún modo en su vocación como historiador y educador?

En mi familia éramos cinco hermanos. Mi papá se convirtió en agricultor, porque antes era mecánico, mientras que mi mamá era ama de casa, lo que era muy típico en los tiempos de entonces. De niño, viví en la localidad de 
Pueblo Nuevo (provincia de Chepén). En la adolescencia, estudié la secundaria en un internado nacional en San Pedro de Lloc (esta última palabra significa 'puñal', en mochica). Pero como vivir dentro era costoso, tuve que vivir fuera del internado, en la casa de una señora, amiga de mis padres. Para entonces, estudiar la secundaria era lo máximo. Muy poca gente accedía a ella. Por ejemplo, en mi pueblo solo seis logramos ir a la secundaria.

\section{¿No todos estudiaban?}

No, ¿sabes por qué? Porque era muy caro. Había que ir a la capital de la provincia para estudiar.

\section{¿Y no habia colegios nacionales?}

Solo había colegio nacional en la capital de la provincia y en la

capital del departamento, por supuesto. Ni siquiera había universidad en Chiclayo. Teníamos que irnos a Trujillo si queríamos estudiar a nivel superior y eso para mí era muy lejos y difícil, por lo costoso que era.

\section{¿Cómo llegó usted a Lima?}

Mi mamá me preguntó al terminar la secundaria qué quería hacer. Yo quería ser médico porque tenía mucho en mí el sentido del servicio. Pero, finalmente, decidí estudiar Educación y me fui a postular a la Cantuta. Para entonces, se tenía que dar tres exámenes: uno en el colegio, luego otro en Trujillo y el tercero en la capital. 


\section{NADA EN LA EDUCACIÓN ESTÁ PERDIDO}

\section{¿Yqué evaluaban?}

El primero, el del colegio, se evaluaba, sobre todo, la vocación, cómo eras tú, las calificaciones, tus valores morales. La evaluación era escrita. Luego, en Trujillo, el examen sí era sobre conocimientos, porque en la Cantuta había la siguiente política: que si eras de provincia, venías a Lima a estudiar y luego regresabas a tu pueblo a enseñar.

\section{Como nuestro programa Nopoki...}

Sí, pero cuando la Cantuta se convirtió en universidad, porque antes era un instituto pedagógico de varones. Cuando la gente ya se vino a quedar en Lima, la universidad se convirtió en una de formación mixta (hombres y mujeres).

En ese tiempo, con respecto a ahora, era muy común que los profesores fueran varones, ¿a qué se debía esto?

Es que en los 60, la mayoría de estudiantes eran varones, puesto que todavía no estaba permitida la educación de la mujer y la Cantuta era el único sitio donde la mujer podía estudiar Educación. Luego, seguía Enfermería o Medicina, donde iban muy pocas mujeres. Ingeniería ni pensar...

\section{Le faltó contarnos sobre el tercer examen...}

Sí, el problema fue que cuando llegué a Lima a dar el tercer examen, ya había pasado la fecha de la evaluación. Como me quedé sin examen, y ya estaba en Lima, empecé a trabajar. Me pasé cinco años trabajando en una farmacia. 
Llegué a dominar todo lo referente a ello: hacer cucharadas, pomadas, conocía todos los productos, incluso ya recetaba, porque hacía a veces de farmacéutico y otras de médico (risas). Incluso, el dueño me animó a estudiar Farmacia, pero yo no quería, quería Educación e ingresé a San Marcos.

\section{Pero ya en la década de los 60 se concebía una reforma educativa. ¿Cómo era la exigencia académica de ese entonces con relación a la de hoy?}

Ah, eso era porque tuvimos a los mejores profesores. En ese tiempo, las universidades más populares eran San Marcos y la Católica. Todavía no existía Villarreal. A la Católica iban solo los pitucos y a San Marcos los que alcanzaban superar los tres exámenes: el primero de letras, el segundo de ciencias y el tercero la entrevista personal, ¡imagínate!

\section{¿Qué maestros significativos ha tenido?}

Como profesores yo tuve a Augusto Salazar Bondy, a Manuel Beltroit. Incluso Arguedas, aunque no fue profesor de mi base porque estaba estudiando sociología en París, pero sabíamos que enseñaba en mi facultad. También me enseñó Alfredo Rebaza Acosta. Recuerdo que Luis Alberto Sánchez, mi profesor de Historia de América, nos llevó a inaugurar la ciudad universitaria. Allí nos hizo una clase especial en medio de los fotógrafos y periodistas.

\section{¿Cómo eran las clases con estos profesores?}

Era como leerte un libro, o quizás dos libros. Me acuerdo por ejemplo, de Salazar Bondy. La clase era a las 8:00 a. m. pero desde las 6:30 a. m. o 7:00 a. m. ya la gente llenaba un salón de 300 alumnos matriculados; compartíamos 


\section{NADA EN LA EDUCACIÓN ESTÁ PERDIDO}

el salón con gente de Historia, Geografía, de Lengua y Literatura, Filosofía y Ciencias Sociales. Eran parte de los estudios generales. Luego, al segundo año, dabas un examen de las asignaturas que llevabas y después pasabas a tu especialidad. Ahí nos enseñó Luis E. Valcárcel y Pulgar Vidal en Geografía. Gente clásica y de primera magnitud. También Emilio Barrantes, quien después hizo la reforma de la educación en la época de Velasco.

\section{¿Cómo era la relación con ellos, con los profesores?}

Era muy directa, similar a la de aquí (en la UCSS). Un alumno te solicita y tú lo atiendes. Lo malo es que acá no tenemos oficina, allá cada profesor tenía su oficina. Por ejemplo, el doctor Luis Felipe Alarco. Era profesor de Metafísica y discípulo de Nicolai Hartmann, un gran exponente de la fenomenología. Había estudiado en Alemania, la mayoría eran formados en el extranjero. Recuerdo que estábamos estudiando Metafísica con él. En aquellos tiempos los cursos eran anuales, llevábamos tres meses y no entendíamos nada. Entonces, un día nos encontramos con unos amigos y quedamos en ir a la oficina del doctor para que nos recomendara algún libro para leer; llegamos a su oficina y nos preguntó cuánto tiempo teníamos en el curso. "Tres meses", le dijimos. "Ah, no, esperen al quinto mes. Recién ahí entenderán” (risas).

\section{¿Qué buscaba San Marcos de esa época?}

Sobre todo investigación, San Marcos editaba libros a montones, ahora han bajado por la cantidad de alumnos que tienen. En ese entonces te graduabas por tesis y si esta destacaba, inmediatamente te llamaban y te publicaban. Había todo un departamento que se encargaba de eso. 
Una de nuestras amigas de promoción ha sido decana y vicerrectora, pero no la hemos visto con el brillo que tenía cuando estábamos en la universidad. La universidad te hacía leer. Acá me dicen, "profesor necesitamos lo manuales ya para irlos viendo". Yo no los recomiendo, porque a los alumnos les das el manual y eso no más leen, no se van a más, por mucho que les ofrezcas más libros. Entonces, yo he decidido hacerles un cuadro sinóptico y les doy bibliografía para que se vayan a la biblioteca, porque las cosas deben saberlas a profundidad.

\section{Profesor, usted ha escrito muchos libros sobre economía, sicología, educación, historia... ¿Qué le ha motivado ser tan prolífico?}

Yo empecé a escribir libros a partir de mis fichas bibliográficas que aprendí a usar en la universidad. Todos mis cursos los tengo clasificados en fichas. En base a ello, empecé a elaborar mis manuales. Por ejemplo, tengo manuales de educación familiar, historia del pensamiento educativo e historia de la pedagogía que me tomó tres años hacerlo y tiene más de 600 páginas. Cuando empecé a trabajar en el Ministerio (de Educación), me encontré con algunos amigos con quienes estudié en San Marcos y escribían libros, de varias especialidades, menos de historia. Ellos me animaron y me buscaron un editor y comencé a elaborar junto con mi esposa.

\section{Por cierto, ¿cómo la conoció?}

Bueno, yo la conocí a ella en la universidad. Ella estudió dos años en la Facultad de Letras, porque le gustaba la literatura y luego se pasó a Educación. Como ella así hicieron varios porque estudiar Educación era (y es) una carrera práctica. Por ejemplo, ahí conocimos a literatos como 


\section{NADA EN LA EDUCACIÓN ESTÁ PERDIDO}

Gregorio Martínez (cuentista), Martín Quintana (autor de textos de literatura), entre otros.

\section{¿Cuáles han sido los grandes bitos que marcaron su vida profesional? ¿Hubo un antes y un después?}

Quizá cuando se publicó mi primer libro Historia del Perú y del mundo. Ahí me di cuenta de que tenía una capacidad de poder realizar más cosas. Ya desde tiempos de mi enseñanza en el colegio Nuestra Señora de los Desamparados (Breña), el director me veía con mis fichas y me preguntaba: “¿Cuándo vas a publicar un libro?”. Pero el problema que se me presentaba era la redacción, porque más sabía de historia y me tuve que apoyar de mis colegas y amigos que estudiaron literatura y me ayudaron mucho. Cuando publiqué, me di cuenta que rindió y pegó. Para que te des cuenta, que gracias a las ganancias de la primera edición de mi libro pude pagar mi casa (risas).

A partir de ello, otros editores me empezaron a buscar. Por ejemplo, Augusto Benavides Estrada, que después se hizo gran amigo mío. Enseñaba geografía y había fundado una editorial llamada Escuela Nueva. No sé si llegaste a escuchar de él.

\section{¡Claro, yo estudié con los libros de Escuela Nueva en el colegio!}

Bueno, él hacía los libros de geografía, además de enseñar en la Cantuta. Recuerdo que un día me llamó por teléfono para que trabaje con él en la elaboración de libros de historia, pero lamentablemente yo ya me había comprometido con otro editor. Posteriormente, hice un curso de especialización en el Instituto Riva-Agüero y, al final, recuerdo que nos quedamos con el padre Armando Nieto y decidimos innovar 
un curso de Geopolítica, que resultó ser una novedad para el Ministerio cuando lo presenté.

\section{Recuerdo que en la década de los 90 se enseñaba ese curso en la secundaria.}

Yo había ayudado un poco a gestar el programa de Geopolítica para el Ministerio. Nos fuimos y nos entrevistamos con el primer ministro de entonces. Con ello se hizo posible la necesidad de escribir un libro de geopolítica para los profesores, por lo que nos pusimos a investigar. Éramos cuatro profesores quienes hicimos el libro y pegó rapidísimo. En cada campaña se vendía miles de ejemplares, que más o menos costaba S/ 30. 00. Es por ello que no me preocupé mucho por enseñar en la universidad, porque vivía cómodamente haciendo libros y vendiéndolos. Pero muchos de mi generación enseñaban en la secundaria y luego en la universidad. Yo no hice carrera en la universidad sino hasta mucho después.

\section{Le be escuchado decir que "la educación es lo único que nos puede salvar”. ¿Cómo ha llegado a esta certeza?}

Llegué a esa conclusión porque veía este país con mucha riqueza, porque somos uno de los pocos países que tenemos una riqueza de clima, de producción, hasta de cultura. Somos creadores de cultura. Según Arnold Toynbee, somos uno de los 10 países que ha dado cultura al mundo. ¿Por qué siendo una cultura así de rica no hemos madurado nunca? Le hemos echado la culpa a la Colonia, pero eso era la afirmación de los indigenistas. Yo me di cuenta, ya cuando estuve en el Ministerio, de que el problema es que quienes han dirigido el país han sido militares, abogados, economistas. Todos ellos creen que la educación es el producto de todo el desarrollo de las 


\section{NADA EN LA EDUCACIÓN ESTÁ PERDIDO}

otras actividades que el hombre tiene, No, para mí la educación es la base de todo el desarrollo. Entonces, el día que desarrollemos una buena educación, el país tendrá que cambiar. Esto es lo que me ha mantenido en la educación.

Otra cosa, cuando hice mi libro de economía, yo afirmé claramente que la educación no es un gasto, es inversión. Ese concepto de gasto lo tiene el Estado, lo tienen los profesores, los padres de familia. No, es una inversión. No saben que es la única cantidad de plata que no se pierde. Por eso debe ser que me quedé en la educación, porque quiero ayudar a la gente, aunque mi sueldo no sea grande. Yo sé que me privo de una ganancia mayor para que un joven gane una beca o media beca. Esa es mi forma de ayudar a la gente porque, si yo pidiera bastante dinero, eso ya no iría en beneficio de algún estudiante. Mira, yo he sido director de colegio, director de instituto, pero cuando llegué a esta universidad, la UCSS, pedí no tener un puesto. Y encontré en esta universidad a una persona que tenía esta misma mirada que yo, que era Andrés Aziani. A él no le gustaban los puestos, quería estar con los alumnos, enseñando, así como yo. Uno aprende dando. Ahí se ve la vocación.

Pero también usted ha enfatizado que dicha educación, que cambia al hombre, debe sostenerse desde una visión cristiana. ¿Cuál es el aporte que solo el cristianismo puede dar para ese gran cambio educativo?

Nosotros tenemos dos fuentes que nos hacen especiales: la fuente nativa que es fuente comunitaria, donde te realizas con la ayuda del otro, $y$ la otra fuente que es el cristianismo, que mira a la persona que trasciende. Este es el valor de nuestra cultura. Estos dos elementos se complementan, porque al estar en una comunidad, ayudas a todos y a través de ellos te ayudas a ti mismo y reconoces tu identidad. Esto solo lo da el cristianismo. Yo estoy leyendo un libro sobre qué es la felicidad según la historia y me 
he encontrado con sorpresas terribles. La gente piensa que la felicidad es su egoísmo. No, la felicidad es proyección hacia afuera. Por eso, el cristianismo reconoce que tú te realizas en cuanto das, más que en recibir. Es la proyección hacia el otro. Esto la gente no lo toma mucho en cuenta.

\section{Usted ha tenido un papel protagónico para que exista un reconocimiento por la figura de Alfredo Rebaza Acosta en Los Olivos. ¿Qué lo motivo a hacerlo?, ¿qué otros héroes “anónimos" conoce y recuerda con mucho cariño?}

El colegio era lo que se denominaba C3 El Trébol, así se llamaba el sistema. Durante ese tiempo se estaba buscando un nombre más apropiado para el colegio y se pensó en darle el nombre de un oficial caído en el conflicto con el Ecuador durante la época de Belaunde. Sin embargo, conversando con mi esposa, que trabajaba ahí, me pregunté por qué no buscar el nombre de un profesor. A mi esposa le gustó la idea y comenzamos a pensar en el nombre de nuestro profesor Rebaza Acosta. Ella se puso en contacto con la familia del profesor para elaborar la parte biográfica y se presentó como parte del expediente. Lo pensamos, sobre todo, por el caso del profesor anónimo que nunca se le reconoce y es el que hace mejor las cosas. Siempre se le reconoce al político, al abogado, un personaje, pero quien experimenta las cosas, quien hace los valores, es el profesor común y corriente. Por eso el valor del reconocimiento, por eso yo admiraba a Andres Aziani, porque buscaba siempre algún servicio.

Otro de los profesores anónimos que recuerdo era Emilio Barrantes, quien fue presidente de la Comisión de Reforma Educativa en la época de Velasco, pero como estaba vinculado con un dictador, fue borrado del mapa del reconocimiento. Otro, para mí, era Luis Alberto Sánchez, que fue el peruano que ha escrito más libros en el Perú (empezó desde los 18 


\section{NADA EN LA EDUCACIÓN ESTÁ PERDIDO}

años). También puedo mencionar a Everardo Zapata Santillana, el creador de Coquito. Es un profesor anónimo que descubrió un método en Arequipa y lo emplea con sus alumnos. Lo publicó ante sus amigos primero, luego en su pueblo y, después, a nivel nacional. Coquito, por ejemplo, es un caso especial porque es el único libro que no menciona al autor y es el único en donde el usuario gasta dos libros al año, porque el niño, antes de los seis meses ya ha deshecho el libro. Y es barato, además.

En su experiencia, ¿por qué cree que la educación peruana no mejora? A pesar de las grandes reformas y cambios curriculares, ¿por qué la educación sigue siendo un desafío permanente?

La primera causa es que en la educación peruana siempre se ha buscado un modelo desde fuera. Y todo lo que es adecuado es peligroso o no favorable. El segundo problema es que la educación siempre ha estado dirigida por la economía o por la política. El político hace su propia reforma educativa cada cambio de gobierno. No lo hace un grupo de profesores. El día que lo haga un grupo de profesores en donde haya filósofos, gestores en currícula, entre otros, cambiará la educación. Incluso, la reforma educativa superior, ¿quién lo hizo? Un militar político que no era educador de formación ni de vocación. El tercer problema es que siempre hemos sujetado la educación a lo económico, cuando es un espejismo que la economía pueda condicionar a la educación. Tú puedes hacer de un niño, pobre y hambriento, una excelente persona.

Lo económico sí te ayuda, te complementa, te empuja, te anima, pero no porque no tengas plata no puedes educarte. Hay mucha gente pobre que ha logrado destacar y hacer de la educación algo importante en su vida. Por ejemplo, uno de los profesores más importantes del país ha 
sido José Encinas. Él era un indígena de Puno que llegó a ser profesor, y después fue ministro de Educación, rector de San Marcos y representante en el parlamento, siendo de origen pobre. Solo él dijo que lo único que puede salvar la democracia es la educación y también que el orgullo más grande de un ciudadano en el Perú es ser profesor o educador.

\section{Usted también ha enfatizado que es importante la historia para aprender} de nuestros errores, pero parece que nos falta, como cultura, seguir aprendiendo de nuestros errores históricos. ¿No será acaso que nos falta educar en una historia brutalmente honesta y menos rosa? La historia del Perú que nos enseñan en los colegios es una historia que no toca temas negativos de nuestra historia pero que son parte de ella.

Es que tomamos los acontecimientos históricos de mala manera, como si fuera un fracaso. No debe ser así, el fracaso debe ser tomado como experiencia, como la educación. Por ejemplo, si un alumno jala, no es fracaso, es experiencia. Lo único que debe hacer es reconocer esta experiencia, analizarla para superarla. La guerra con Chile nos debió haber traído experiencias para no volverlas a repetir. Estas experiencias son las que hacen a los pueblos. Pero no, nosotros nos olvidamos de esto y lo tenemos como fracaso y queremos olvidar y volvemos a repetir los mismos hechos. El pueblo que supera estas experiencias es el que llega a superar también hechos históricos. No estudiamos la historia como debería ser, teniendo en cuenta la coyuntura, la estructura y acontecimiento. Nosotros lo estudiamos de manera lineal y solo estudiamos guerras, personajes, presidentes, héroes. Solo vemos la parte negativa, de la muerte, pero no asimilamos los valores en la experiencia. No tomamos conciencia de lo que hemos sido. 


\section{Ahora cuéntenos, ¿desde cuándo vive en Lima Norte?, ¿qué lecciones le ha enseñado esta vida en este particular lugar?}

Yo vivo aquí en Los Olivos desde 1974, cuando todavía pertenecía a San Martín de Porres. Lima Norte es la parte de Lima que más ha progresado y no porque vinieron los grandes capitalistas y se aislaron del mundo como en el distrito de Asia; no progresaron porque se juntaron los ricos en un barrio, no. Aquí se progresó porque vino gente de trabajo, gente que quería mejorar, como pioneros, que se fijan en el progreso. Por ejemplo, la urbanización de Pro es un modelo especial arquitectónico, que vienen a estudiarla. Está categorizado como una estructura especial. ¿Quién sabe eso? Solo la educación te hace valorar esto. Aquí, en Lima Norte la gente es pujante, quieren mejorar, quieren ser mejores, hacer que sus hijos crezcan, y esa ha sido la brillante idea que tuvo la Universidad Católica Sedes Sapientiae para descubrir cómo esta gente necesitaba — nada más— el empuje de la educación. Eso está demostrando la teoría que el empuje de la educación hace de una ciudad, pueblo, cultura, barrio, de una zona o de una familia, una transformación completa.

Usted ha formado parte de la historia del colegio El Buen Pastory de la UCSS. Después de más de 20 años de crecimiento, ¿qué frutos ha identificado?

Cuando yo vivía acá, Comas era un conjunto de establos, en donde la gente había invadido el cerro y en donde guardaban muchas cosas y también criaban animales. Te imaginas a un hombre simple y corriente que no tiene conciencia de lo que tiene y tiene una casa de mil metros. Si tú educas a su hijo y le haces ver que tiene una riqueza botada allí; si le das educación universitaria, le das una categoría de educación superior y le das 
una categoría de invención a través de la creación de empresas, esos mil metros se transformarán en una riqueza incalculable. Eso es lo que hizo esta universidad en primer lugar, despertó la capacidad del ser humano a través de la educación. La universidad fue el centro de la cultura que transformó la forma de pensar de todos.

En segundo lugar, la Iglesia se ha dado cuenta de algo muy importante, ya no basta con darle al joven catequesis, ni regalos en la parroquia. La riqueza más grande que le puedes dar es la educación. La gran mayoría de gente que viene a estudiar es de parroquias, a diferencia de otras universidades. Es por eso que en esta universidad hay otra mirada, porque le creas un sentido de comunidad que ojalá no se pierda. Acá te forman para que tengas un título, para que ayudes y progreses con y para otros. Ese es el valor de la educación: que te cambien de una mentalidad consumista y egoísta a preguntarte ¿quién eres? El tener ingresos o pensar en un doctorado es consecuencia de este esfuerzo.

\section{Finalmente, ¿le gustaría dar un mensaje a los jóvenes universitarios? \\ La educación no es un gasto, es una inversión. Nada en la educación está perdido. La educación es la única posesión que te incrementa, te da riqueza y lo puedes llevar a donde sea. Es lo único que te puede salvar de los males del mundo, hasta de ti mismo; y, por supuesto, con ello también se puede realizar tu vocación cristiana.}

